\title{
Two-Dimensional Time-Domain Antenna Arrays for Optimum Steerable Energy Pattern with Low Side Lobes
}

\author{
Alberto Reyna, ${ }^{1,2}$ Marco A. Panduro, ${ }^{2}$ Gerardo Romero, ${ }^{2}$ Juvenal Villanueva, ${ }^{1}$ \\ Fernando F. Rivera, ${ }^{1}$ and Aldo L. Méndez ${ }^{2}$ \\ ${ }^{1}$ Sistemas Industriales Automatizados, Plaza Vista Hermosa, 88779 Reynosa, Tamp., Mexico \\ ${ }^{2}$ Universidad Autónoma de Tamaulipas UAMR-R, Carretera Reynosa-San Fernando, 88779 Reynosa, Tamp., Mexico \\ Correspondence should be addressed to Alberto Reyna; alberto.reynam@outlook.com
}

Received 10 December 2013; Accepted 6 June 2014; Published 2 July 2014

Academic Editor: Z. N. Chen

Copyright (C) 2014 Alberto Reyna et al. This is an open access article distributed under the Creative Commons Attribution License, which permits unrestricted use, distribution, and reproduction in any medium, provided the original work is properly cited.

\begin{abstract}
This document presents the synthesis of different two-dimensional time-domain antenna arrays for steerable energy patterns with side lobe levels. The research is focused on the uniform and nonuniform distributions of true-time exciting delays and positions of antenna elements. The uniform square array, random array, uniform concentric ring array, and rotated nonuniform concentric ring array geometries are particularly studied. These geometries are synthesized by using the well-known sequential quadratic programming. The synthesis regards the optimal true-time exciting delays and optimal positions of pulsed antenna elements. The results show the capabilities of the different antenna arrays to steer the beam in their energy pattern in time domain and how their performance is in frequency domain after the synthesis in time domain.
\end{abstract}

\section{Introduction}

Historically, the frequency domain antenna arrays have been widely developed for multibeam, shaped, and phased patterns in narrowband applications. Lately, modern requirements lead to the use of ultrawideband (UWB) technology for high rate data transmission and high sensitivity $[1,2]$. The UWB arrays can be the solution for such requirements; however, the performance of the power pattern varies with the frequency. Hence, the synthesis of an UWB array is very difficult because of a frequency domain perspective. An alternative way for this type of synthesis is to view the problem from the timedomain perspective where it could be solved in an easier way. In this case, time-domain (TD) or pulsed antenna arrays have recently been studied by regarding their advantages of low side lobes and high resolution for the modern applications such as radar, remote sensing, and communications [3]. Previous works in the literature include studies mainly for the TD linear arrays, exploiting its features of fixed and scanning beam of patterns [4-13]. The research in [14] addresses a study of performance of large two-dimensional time-domain (TDTD) antenna arrays for a very narrow-fixed pattern in imaging applications. Moreover, the TD circular arrays have been also studied by modeling Hermite-Rodriguez waveforms and a generalized hypergeometric function for a broadside-fixed pattern [15]. The performance tradeoffs associated with the selection, size, and distributions of time delays in large TD uniform square arrays were presented in [16]. So far, the performance of TDTD antenna arrays for an optimum steerable energy pattern (SEP) is an open research field. Here, the authors present a novel study of the uniform and nonuniform TDTD antenna arrays geometries, particularly conventional uniform square array (CUSA), conventional concentric ring array (CCRA), uniform square array (USA), random array (RA), uniform concentric ring array (UCRA), and rotated nonuniform concentric ring array (RNUCRA). The study considers the synthesis of different geometries for a SEP with low side lobe level (SLL). To this end, the well-known sequential quadratic programming (SQP) [17] is utilized by finding out the optimal true-time exciting delays and optimal positions of pulsed antenna elements. This algorithm has been recently proved to be very efficient in the synthesis of antenna arrays [18-21]. We do not claim SQP is the best-suited algorithm to synthesize TDTD 
antenna arrays. The selection of the best-suited algorithm is still an open research field. Hence, the novelties of this research are (1) a study of the performance of different TDTD antenna arrays for an optimum SEP by means of a synthesis based on SQP and (2) a study of the performance of the patterns in frequency domain for the best antenna array geometries after the synthesis in time domain.

\section{Time-Domain Antenna Arrays Models}

Let us consider the TDTD antenna arrays as a group of $N$ pulsed antenna elements located on a plane as shown in Figure 1. Each antenna is receiving (or radiating) a time short pulse in each direction. The short pulses are added with different time delays resulting in a far-field waveform. Its distribution depends on four parameters which are the amplitudes, the antenna positions, the waveform of the short pulses, and the time delays among the short pulses $[1,3]$.

Let us assume the 5th derivative Gaussian pulse as the waveform of the receiving short pulse for each direction as follows [22]:

$$
f_{n}(t)=A\left(-\frac{t^{5}}{\sqrt{2 \pi} \sigma^{11}}+\frac{10 t^{3}}{\sqrt{2 \pi} \sigma^{9}}-\frac{15 t}{\sqrt{2 \pi} \sigma^{7}}\right) \exp \left(-\frac{t^{2}}{2 \sigma^{2}}\right)
$$

where $A=1$ and $\sigma=51 \mathrm{ps}$ are chosen to satisfy the FCC regulations [22-25]. Now, the antenna array can be modeled for uniform or nonuniform distributions of the antenna positions and time delays so that the far-field waveform can considerably change. This gives us the opportunity to modify the radiation properties of the TDTD antenna array. In this case, it is preferable to take the energy pattern into account since it represents the behavior of the power pattern in the corresponding spectrum. Hence, the energy pattern is defined as [26]

$$
P_{\text {energy }}(\theta, \phi)=\int_{0}^{t}|s(t, \theta, \varphi)|^{2} d t,
$$

where the $s(t, \theta, \varphi)$ is the far-field waveform of the antenna array as specified in Figure 1. The spectrum in frequency of $s(t, \theta, \varphi)$ is obtained by a Fourier transformation as follows:

$$
P\left(f_{r}, \theta, \varphi\right)=\left|\int_{-\infty}^{\infty} s(t, \theta, \varphi) e^{-j 2 \pi f_{r} t} d t\right|,
$$

where $f_{r}$ is the frequency from $3.1 \mathrm{GHz}$ to $10.6 \mathrm{GHz}$ according to the FCC regulations for an UWB system [22-25]. The formulations of the far-field waveform $s(t, \theta, \varphi)$ for the USA, RA, UCRA, and RNUCRA geometries are detailed in the next subsections.

2.1. Uniform Square and Random Array. Firstly, consider $N$ pulsed antennas in the plane $X-Y$; the far-field waveform is [27]

$$
s(t, \theta, \varphi)=\sum_{n=1}^{N} a_{n} f_{n}\left(t-\Delta t+\tau_{p}-\tau_{n}, \theta, \varphi\right),
$$

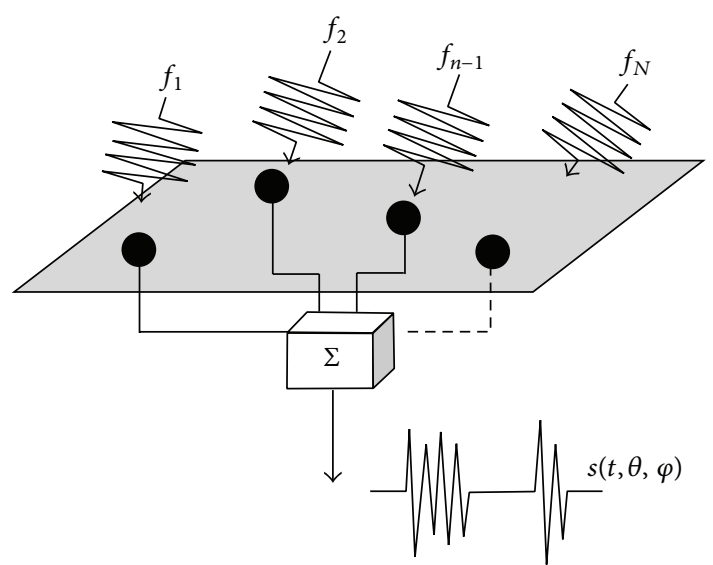

FIGURE 1: Two-dimensional time-domain antenna array.

where

$$
\begin{gathered}
\Delta t=\frac{x_{n}}{c} \sin \theta \cos \varphi+\frac{y_{n}}{c} \sin \theta \sin \varphi, \\
\tau_{p}=\frac{x_{n}}{c} \sin \theta_{0} \cos \varphi_{0}+\frac{y_{n}}{c} \sin \theta_{0} \sin \varphi_{0}, \\
\tau_{n} \in\left[0, \tau_{\max }\right] .
\end{gathered}
$$

The term $\left(x_{n}, y_{n}\right)$ is the $n$th antenna element position in the plane $X-Y$. The direction $(\theta, \varphi)$ represents the elevation and azimuth angles and $\left(\theta_{0}, \varphi_{0}\right)$ is the maximum direction. The constant $c$ is the light speed. The $\tau_{p}$ and $\tau_{n}$ are the progressive and disturbed exciting time delays of the $n$th antenna element, respectively. The $\tau_{\max }$ is the maximum value of exciting time delays. And $a_{n}$ is the amplitude of the $n$th antenna element. For this model, we propose two synthesis cases: (1) a USA with equally spaced antennas and symmetric-disturbed exciting delays and (2) a RA with null disturbed exciting delays and random positions of antenna elements.

2.2. Uniform and Nonuniform Concentric Ring Array. Secondly, consider $N$ pulsed antennas grouped in $N_{r}$ rings in the plane $X-Y$; the far-field waveform is

$$
\begin{aligned}
s(t, \theta, \varphi)= & a_{c} f\left(t-\tau_{c}, \theta, \varphi\right) \\
& +\sum_{m=1}^{N_{r}} \sum_{n=1}^{N_{m}} a_{n m} f\left(t-\Delta t+\tau_{p}-\tau_{n m}, \theta, \varphi\right),
\end{aligned}
$$

where

$$
\begin{gathered}
\Delta t=\frac{r_{m}}{c} \sin \theta \cos \left(\gamma_{n m}-\varphi\right), \quad \gamma_{n m}=\sum_{n=1}^{N_{m}}\left(\frac{2 \pi n}{N_{m}}+\beta_{m}\right) \\
\tau_{p}=\frac{r_{m}}{c} \sin \theta_{0} \cos \left(\gamma_{n m}-\varphi_{0}\right) \\
\tau_{n m} \in\left[0, \tau_{\max }\right] .
\end{gathered}
$$

The term $N_{m}$ is the number of antennas in the ring $m$ and $r_{m}$ is the radius of the $m$ th ring. The angle $\gamma_{n m}$ is the angular 
position of the element $n$ in the ring $m ; \tau_{n m}$ and $\tau_{c}$ are the disturbed exciting delay of the element $n$ in the ring $m$ and the disturbed exciting delay of the central element, respectively. The term $\beta_{m}$ is a rotation angle for the ring $m$. And $a_{c}$ and $a_{n m}$ are the amplitudes for the central element and the $n$th antenna element in the ring $m$, respectively. We also propose two synthesis cases: (1) a UCRA with equally spaced rings, null rotation angles, and disturbed exciting delays and (2) a RNUCRA with null disturbed exciting delays, nonuniform radii of the rings, and nonuniform rotation angles.

\section{Problem Statement}

The synthesis problem consists in obtaining a SEP with low side lobe level by using different geometries of TDTD antenna arrays. We propose the next objective function:

$$
\begin{aligned}
& \text { of }(z) \\
& =\sum_{m=1}^{N_{D}}\left(P_{\text {energy }}\left(\theta_{\text {SLL }}, \varphi_{\text {SLL }}\right)+\left(\theta_{0 m}-\theta_{\text {obt }}\right)+\left(\varphi_{0 m}-\varphi_{\text {obt }}\right)\right) .
\end{aligned}
$$

The $z$ is the vector of the design variables. The content of this vector depends upon the TDTD antenna array geometry, that is, disturbed exciting delays for uniform geometries (USA and UCRA) and antenna positions for nonuniform geometries (RA and RNUCRA). The term $P_{\text {energy }}\left(\theta_{\text {SLL }}, \varphi_{\text {SLL }}\right)$ is the maximum side lobe level and $\left(\theta_{\mathrm{obt}}, \varphi_{\mathrm{obt}}\right)$ is the maximum direction of the energy pattern that is obtained in each iteration. The objective function regards $N_{D}$ directions of the main beam. The $\left(\theta_{0 m}, \varphi_{0 m}\right)$ is the desired main lobe direction. To solve this nonlinear problem, our procedure consists in minimizing the above objective function by using the SQP algorithm which is described in the next subsection.

3.1. Sequential Quadratic Programming. We opted to use the SQP algorithm as an approach for the synthesis of the TDTD antenna arrays for its good performance in solving constrained optimization problems with nonlinear multivariable functions. The efficiency of this algorithm has been demonstrated in different antenna optimization problems as in [18-21]. Here, the SQP algorithm considers the nonlinear problem as follows:

$$
\min _{z} \text { of }(z) \quad \text { such that }\left\{\begin{array}{l}
c_{i}(z) \leq 0, \\
z_{\min } \leq z \leq z_{\max } .
\end{array} \quad i \in\{1,2, \ldots, I\}\right.
$$

The $c_{i}(z)$ is the $i$ th nonlinear constraint function. The $I$ is the maximum number of nonlinear constraints. The $z_{\min }$ and $z_{\max }$ are the limits of the search space for each element of the vector $z$. The SQP algorithm solves the above nonlinear problem by using a succession of quadratic programming subproblems. The statement for these subproblems is the following:

$$
\begin{gathered}
\min _{d} \operatorname{of}_{s}(d): C(z)+A(z)^{T} d \leq 0, \quad i \in\{1,2, \ldots, I\} \\
\operatorname{of}_{s}(d)=\nabla(\text { of }(z))^{T} d+\frac{1}{2} d^{T} H d \\
C(z)=\left\{c_{1}(z), c_{2}(z), \ldots, c_{I}(z)\right\}^{T} \\
A(z)=\left\{\nabla c_{1}(z), \nabla c_{2}(z), \ldots, \nabla c_{I}(z)\right\}^{T}
\end{gathered}
$$

where the of $(d)$ is the objective function of each subproblem. This function is a quadratic approximation to the Lagrangian function [28]. The constraints of each subproblem are linearizations of the constraints in the original nonlinear problem. And the $H$ is a Hessian approximation of the Lagrangian function of the objective function [21]. In order to solve a constrained nonlinear problem, the SQP algorithm iteratively obtains the solution $x_{k}$ in the direction $d_{k}$ at the iteration $k$, where the $d_{k}$ is the search direction vector which represents the solution of the subproblem defined in (10). The solutions for the next generation $\left(z_{k+1}, d_{k+1}\right)$ are obtained by the current solution $x_{k}$ in the direction $d_{k}$ :

$$
z_{k+1}=z_{k}+\mu_{p} d_{k}
$$

where $\mu_{p} \in[0,1]$ is a suitable step length parameter. Then, the algorithm converges toward the optimal solution $\left(z_{k}^{*}, d_{k}^{*}\right)$ by solving the quadratic programming subproblems defined in (10). In the next section, the obtained results are described by applying this algorithm in the synthesis of TDTD antenna arrays.

\section{Simulation Results}

The well-known SQP is applied to optimize different antenna array geometries with similar configuration as in [21]. The SEP is targeted to $N_{D}=64$ directions by considering the $\theta_{0}=\left\{15^{\circ}, 25^{\circ}, 35^{\circ}, 45^{\circ}, 55^{\circ}, 65^{\circ}, 75^{\circ}, 85^{\circ}\right\}$ and $\varphi_{0}=$ $\left\{15^{\circ}, 25^{\circ}, 35^{\circ}, 45^{\circ}, 55^{\circ}, 65^{\circ}, 75^{\circ}, 85^{\circ}\right\}$ for the synthesis process. We consider only 64 directions as a discretization of the infinite number of directions in the space within the synthesis process. Moreover, we consider $N=16$ elements for the different geometries in order to do a fair comparison. Their amplitudes are uniform for all the geometries. Figures 2, 3, 4, and 5 show the obtained results for USA, RA, UCRA, and RNUCRA, respectively. In these Figures we present (a) the positions and arrangement of disturbed exciting delays for the optimized SEPs; each color represents a different disturbed exciting delay, (b) the SEP targeted to the direction $\theta_{0}=\varphi_{0}=45^{\circ}$, (c) the main cuts in azimuth during steering beam with $\theta_{0}=45^{\circ}$, and (d) the main cuts in elevation during steering beam with $\varphi_{0}=45^{\circ}$. It shows sweeps of the main beam in azimuth and elevation planes for a better illustration.

The USA geometry utilizes four different disturbed exciting delays in Figure 2. The value of these delays is in accordance with a maximum of $\tau_{\max }=100 \mathrm{ps}$. The uniform spacing is fixed among the antenna elements with a value of 


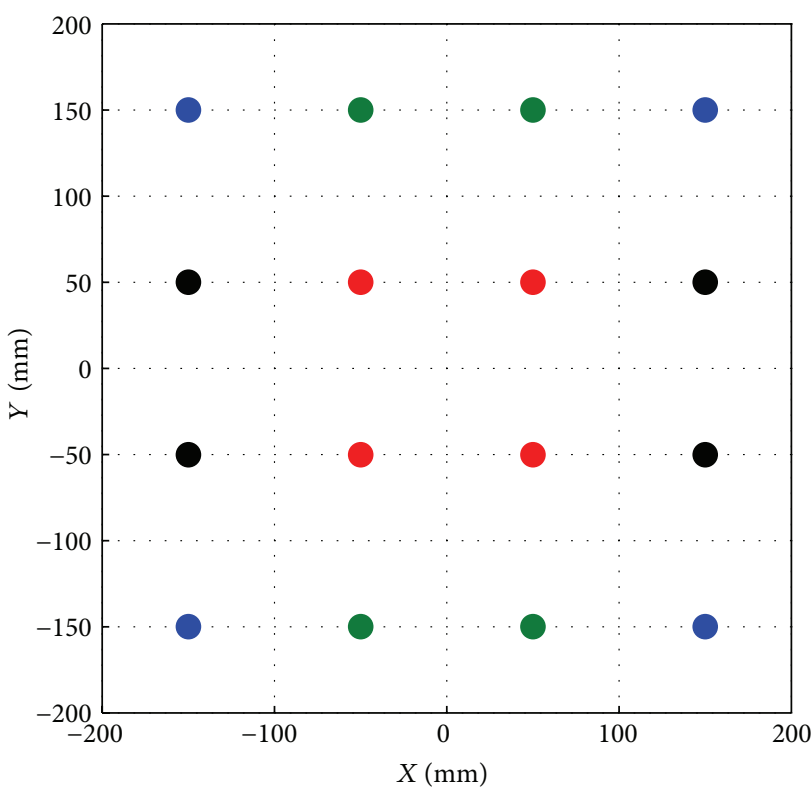

(a)

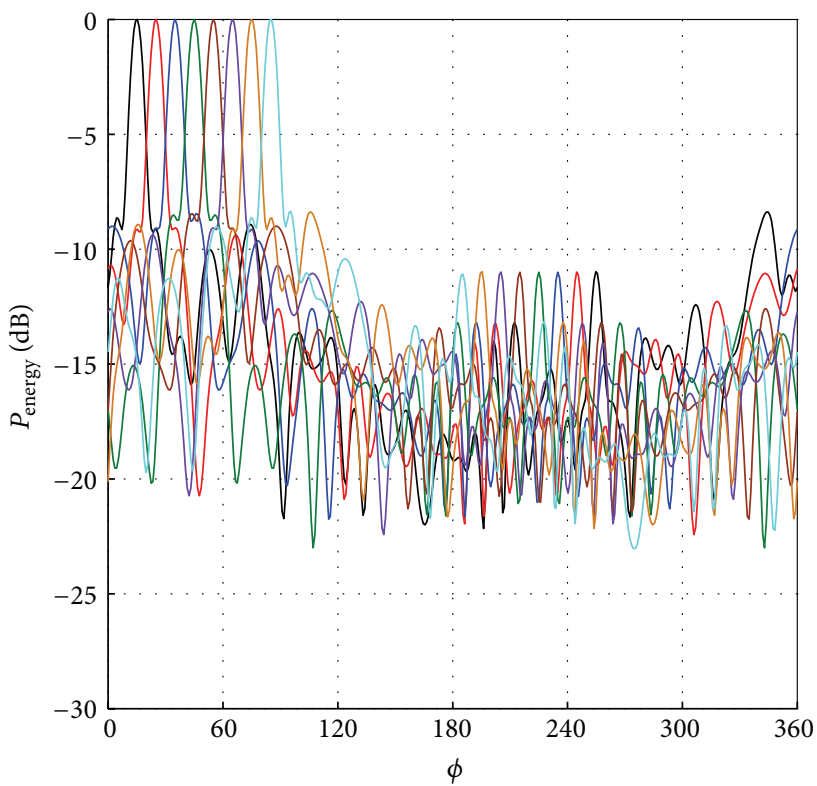

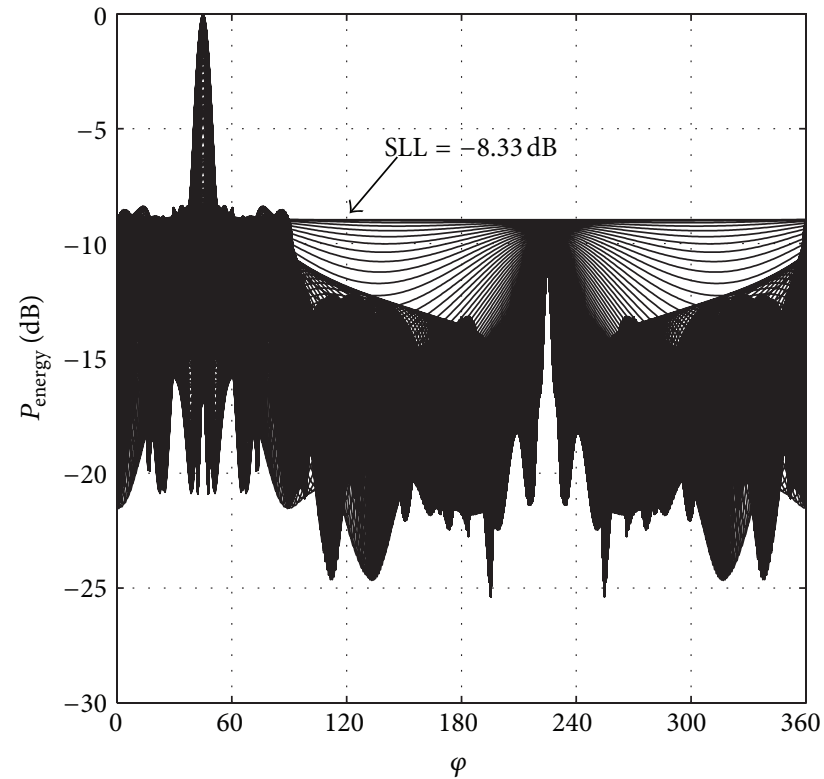

(b)

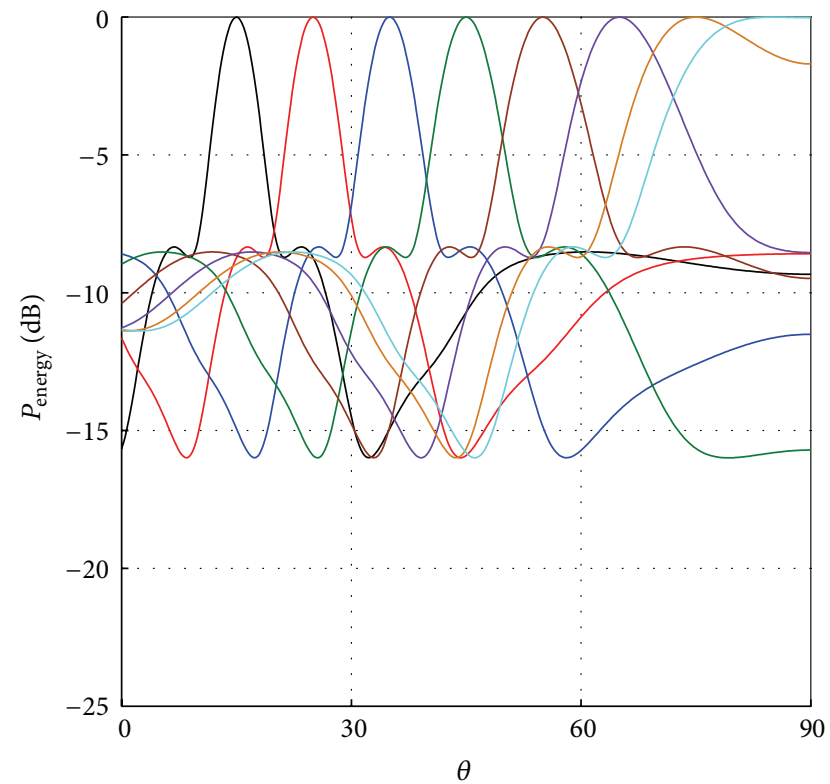

$\begin{aligned}-\theta_{0} & =15^{\circ} & -\theta_{0} & =55^{\circ} \\ \theta_{0} & =25^{\circ} & -\theta_{0} & =65^{\circ} \\ -\theta_{0} & =35^{\circ} & -\theta_{0} & =75^{\circ} \\ -\theta_{0} & =45^{\circ} & -\theta_{0} & =85^{\circ}\end{aligned}$

(d)

Figure 2: Result of USA: (a) antenna and delay distributions, (b) SEP in $\theta_{0}=\varphi_{0}=45^{\circ}$, (c) azimuth cuts with $\theta_{0}=45^{\circ}$, and (d) elevation cuts with $\varphi_{0}=45^{\circ}$.

$100 \mathrm{~mm}$. This value can be enough to avoid serious coupling effects according to [4]. The performance of this geometry permits a SLL $=-8.33 \mathrm{~dB}$ during the steering beam.

The RA shown in Figure 3 is an alternative geometry that can perform the SEP with null disturbed exciting delays. This geometry can permit a better performance of the SEP with a
SLL $=-10.45 \mathrm{~dB}$ during the steering beam. This is achieved by increasing slightly the beamwidth in the search of the optimum solution within the synthesis process. We restrict the minimum spacing among the elements to $50 \mathrm{~mm}$. This can also be enough to avoid serious coupling effects according to [5]. 


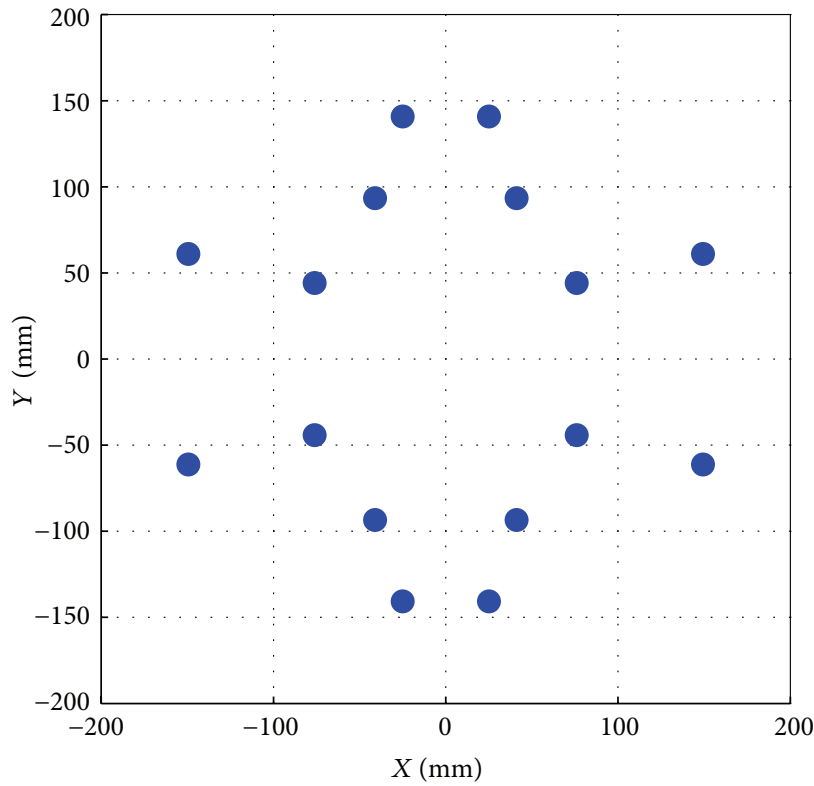

(a)
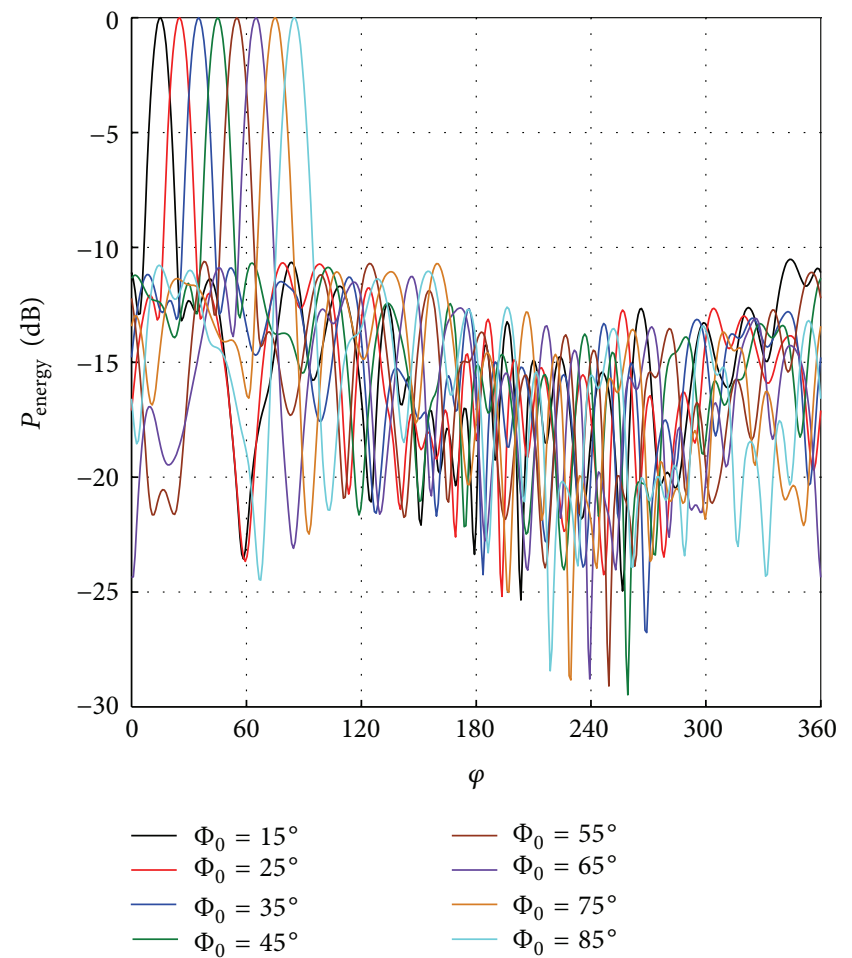

(c)

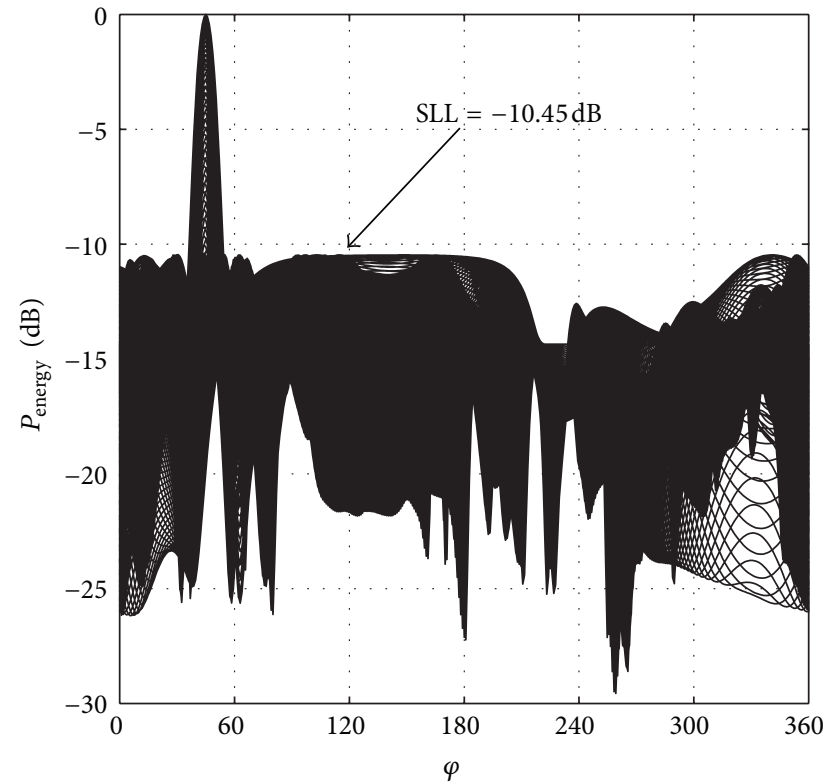

(b)

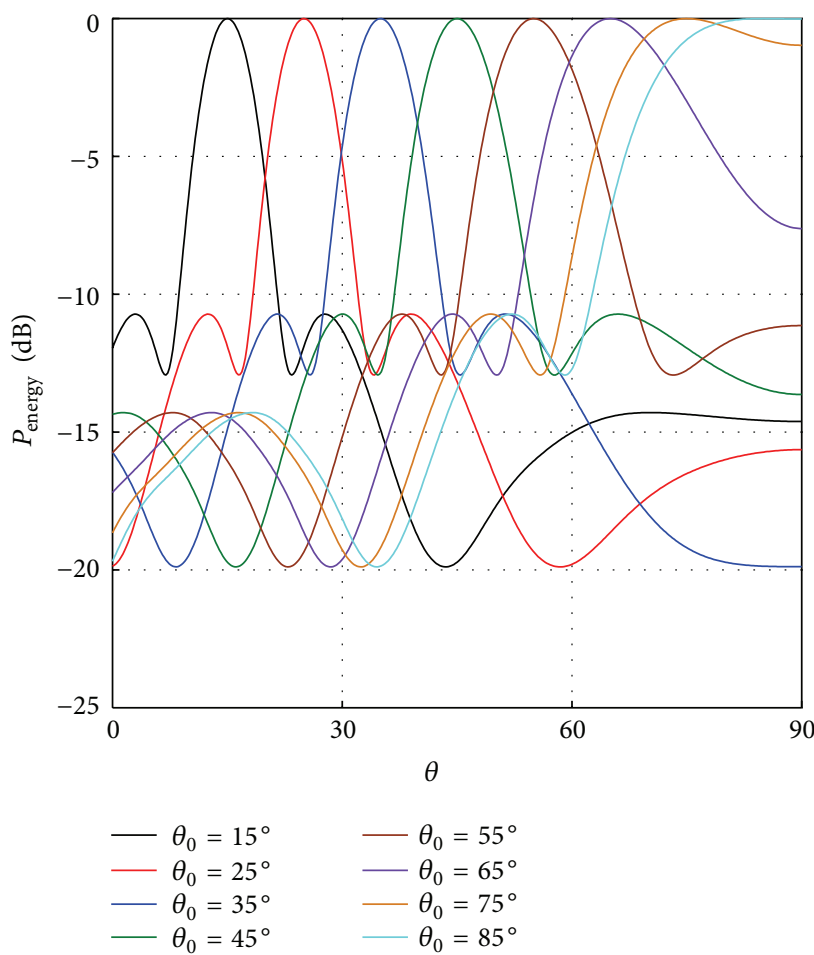

(d)

FIGURE 3: Result of RA: (a) antenna and delay distributions, (b) SEP in $\theta_{0}=\varphi_{0}=45^{\circ}$, (c) azimuth cuts with $\theta_{0}=45^{\circ}$, and (d) elevation cuts with $\varphi_{0}=45^{\circ}$. 


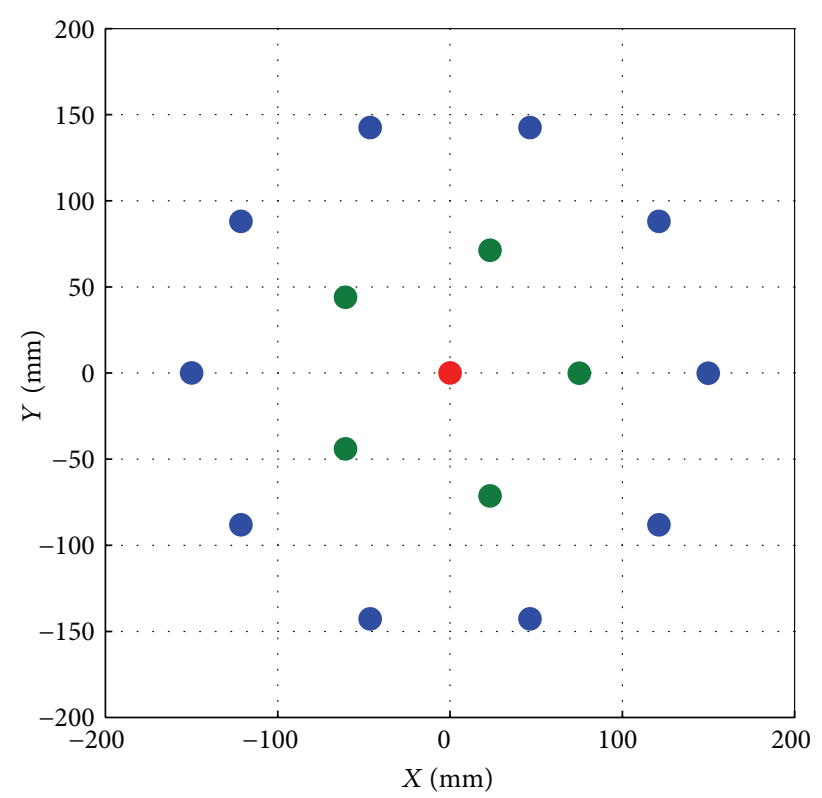

(a)

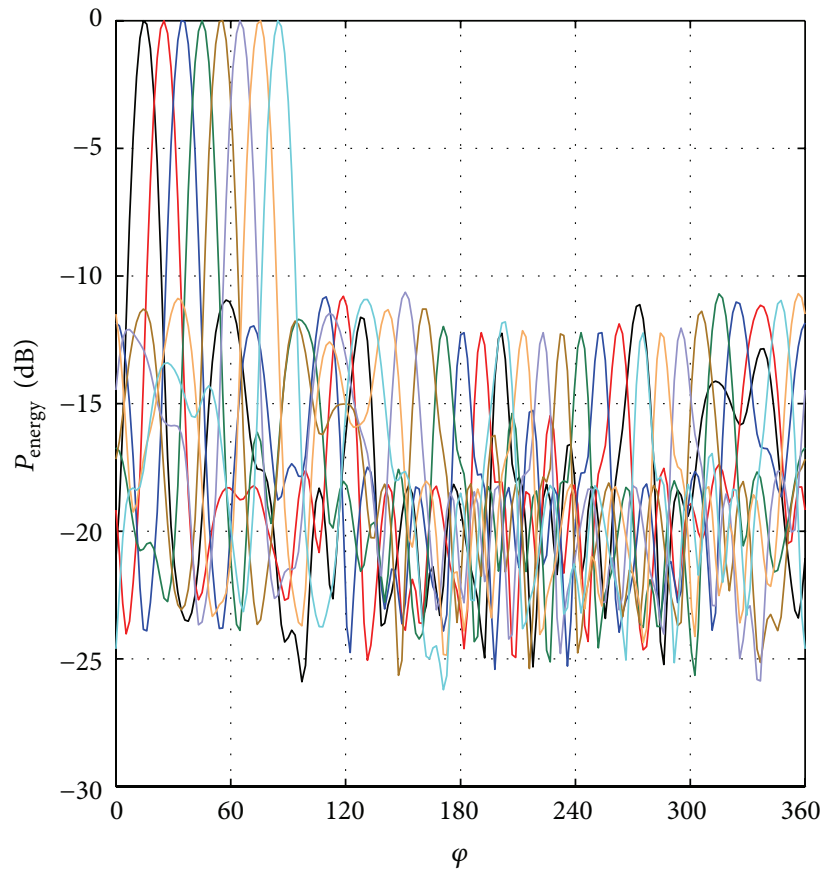

(c)

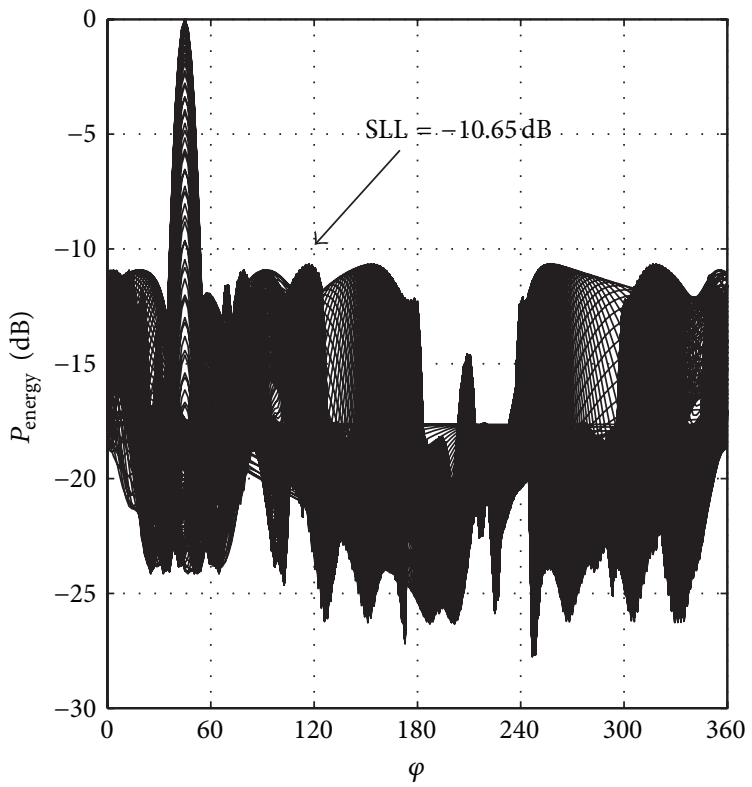

(b)

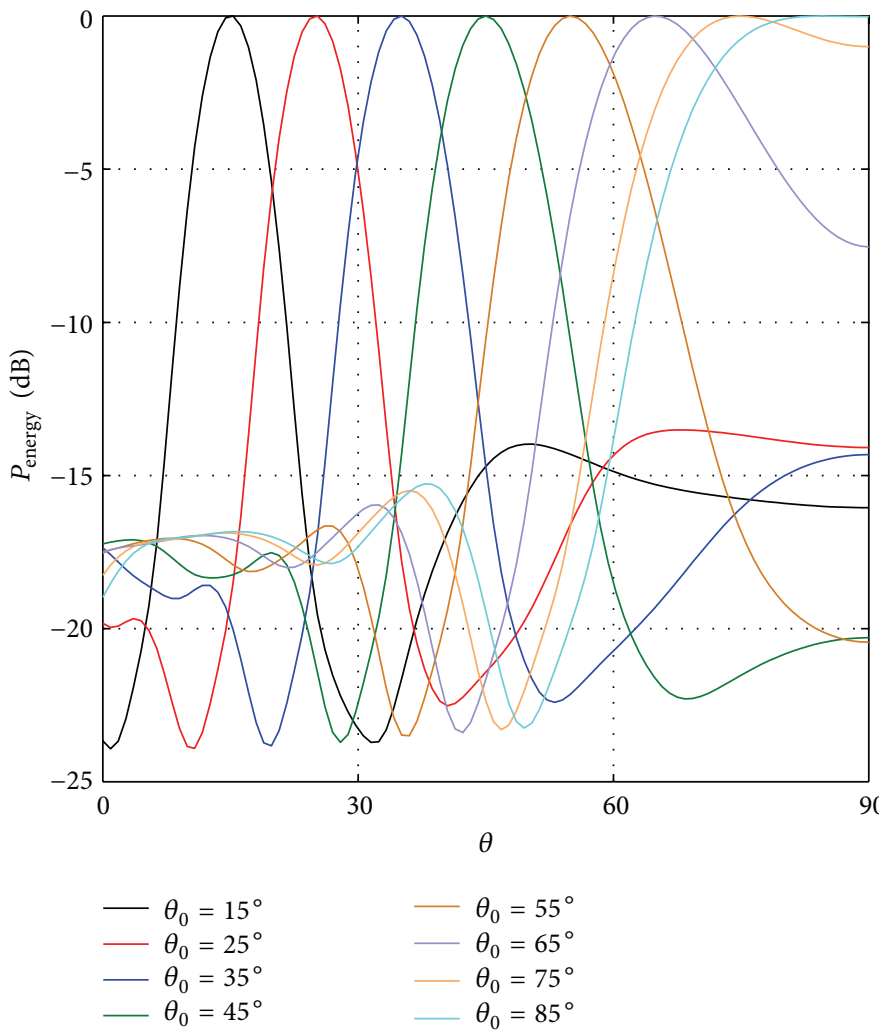

(d)

Figure 4: Result of UCRA: (a) antenna and delay distributions, (b) SEP in $\theta_{0}=\varphi_{0}=45^{\circ}$, (c) azimuth cuts with $\theta_{0}=45^{\circ}$, and (d) elevation cuts with $\varphi_{0}=45^{\circ}$.

Figure 4 shows the SEP of UCRA. This synthesis resulted in a value of SLL $=-10.65 \mathrm{~dB}$. An interesting behavior of this geometry is a considerable reduction of the energy in the opposite direction of the main beam as shown in Figure 4(b).
This geometry utilizes a different disturbed exciting delay per ring. The radii of the rings are fixed as $r_{m}=\{75 \mathrm{~mm}, 150 \mathrm{~mm}\}$ with null rotation angles $\beta_{m}=\{0,0\}$ which are enough to avoid serious coupling effects $[4,5]$. 


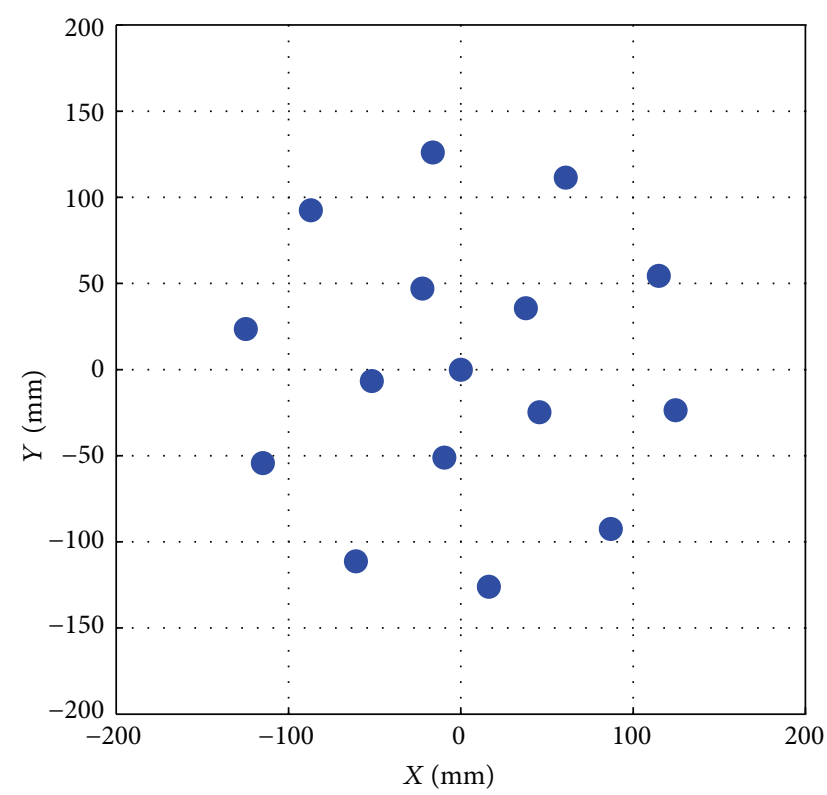

(a)

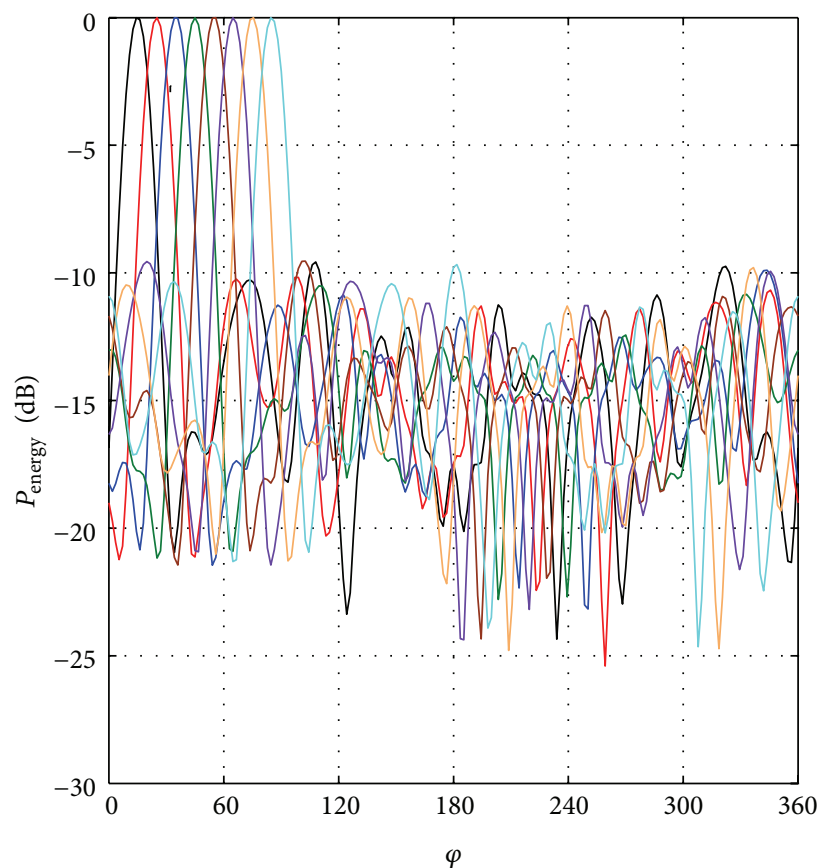
$-\varphi_{0}=15^{\circ}$
$-\varphi_{0}=25^{\circ}$
$-\varphi_{0}=35^{\circ}$
$-\varphi_{0}=45^{\circ}$

$$
\begin{aligned}
-\varphi_{0} & =55^{\circ} \\
-\varphi_{0} & =65^{\circ} \\
-\varphi_{0} & =75^{\circ}
\end{aligned}
$$

(c)

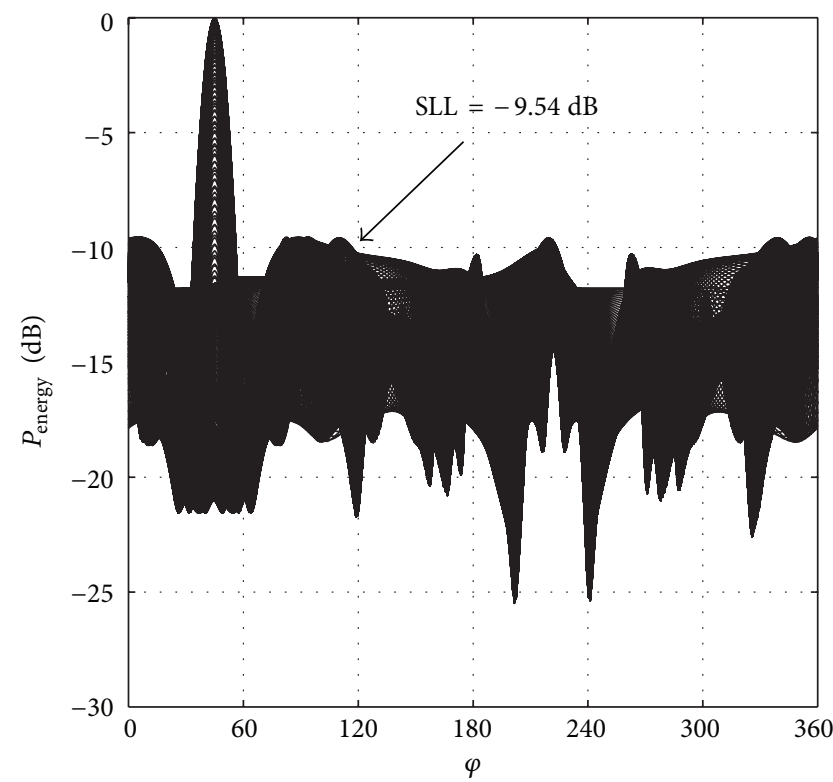

(b)

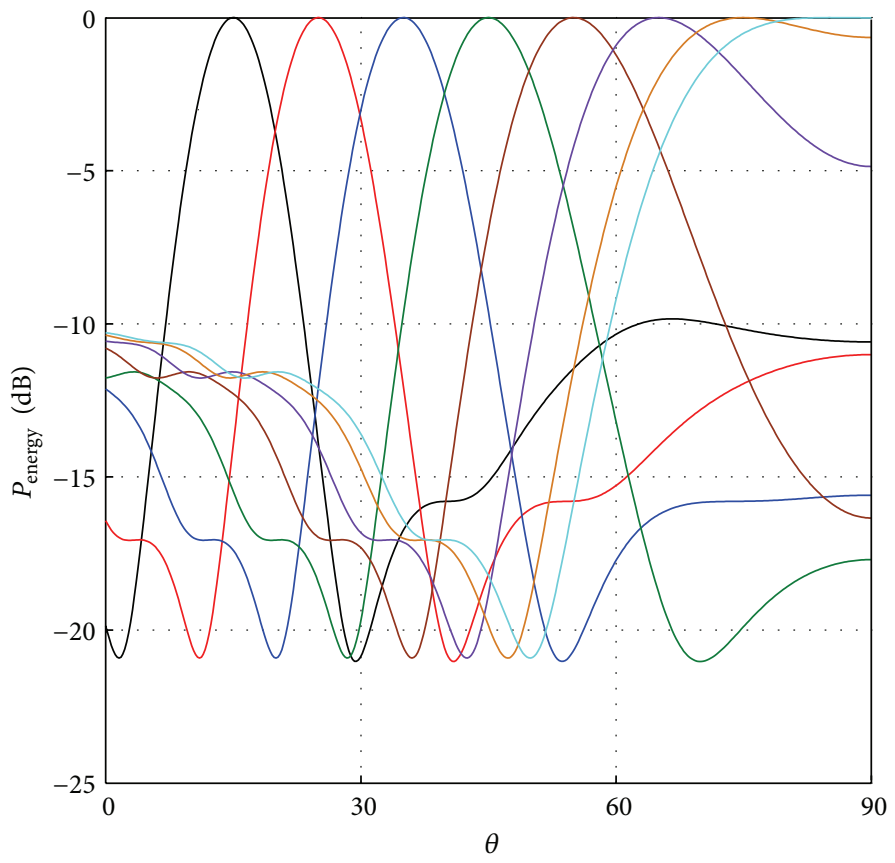

$$
\begin{aligned}
-\theta_{0} & =15^{\circ} & -\theta_{0} & =55^{\circ} \\
-\theta_{0} & =25^{\circ} & -\theta_{0} & =65^{\circ} \\
-\theta_{0} & =35^{\circ} & -\theta_{0} & =75^{\circ} \\
-\theta_{0} & =45^{\circ} & -\theta_{0} & =85^{\circ}
\end{aligned}
$$

(d)

FIGURE 5: Result of RNUCRA: (a) antenna and delay distributions, (b) SEP in $\theta_{0}=\varphi_{0}=45^{\circ}$, (c) azimuth cuts with $\theta_{0}=45^{\circ}$, and (d) elevation cuts with $\varphi_{0}=45^{\circ}$. 


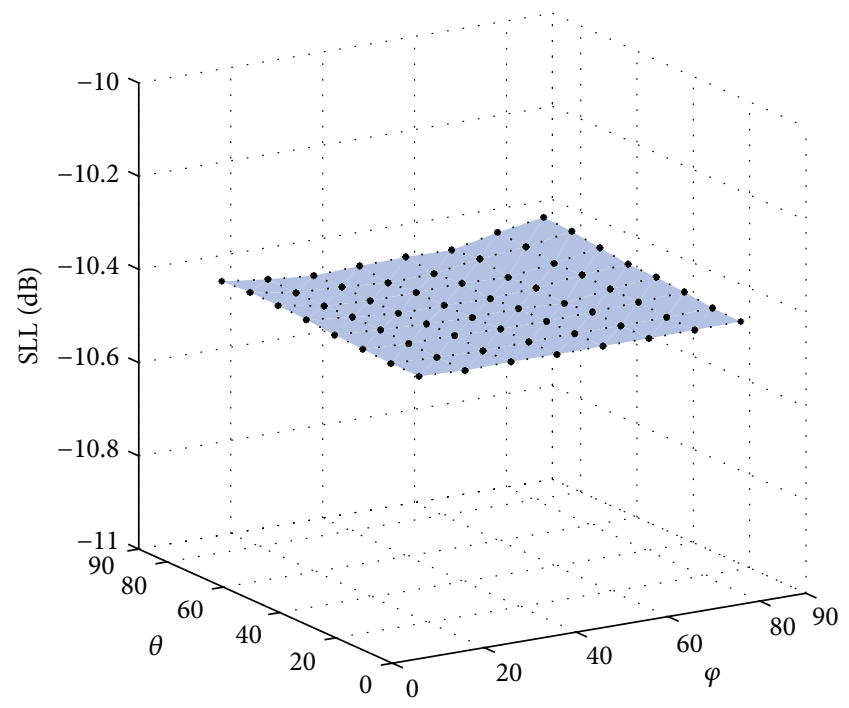

(a)

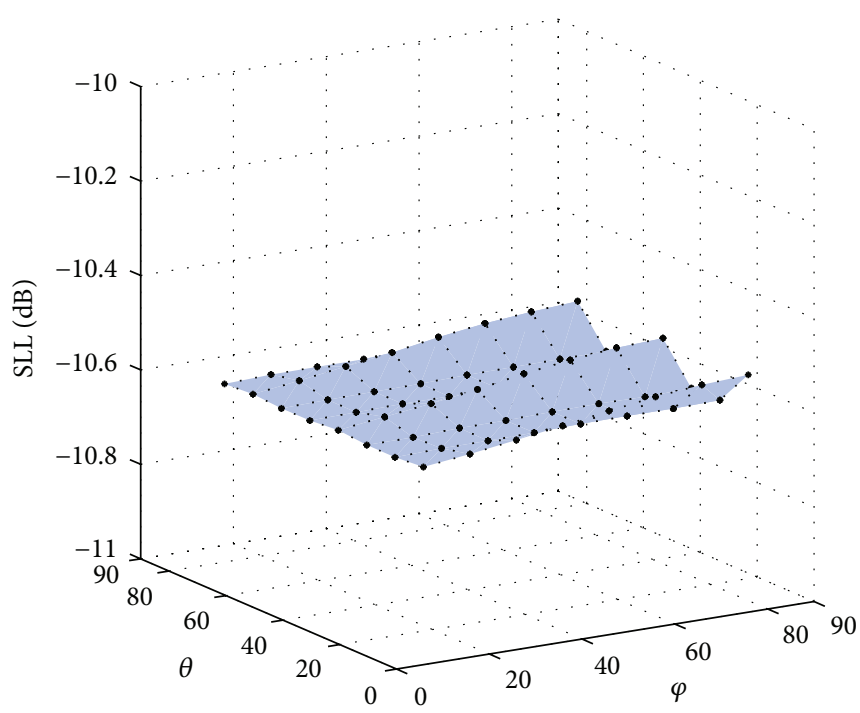

(b)

FIGURE 6: Behavior of peak SLL when the main beam is steered in the optimized directions for (a) RA and (b) UCRA.

The RNUCRA geometry in Figure 5 performs the SEP with SLL $=-9.54 \mathrm{~dB}$ during the steering beam. It utilizes null disturbed exciting delays and nonuniform spacing among the rings. The radii of the rings are restricted in the range of $r_{m} \in[50 \mathrm{~mm}, 75 \mathrm{~mm}]$ in order to avoid serious coupling effects [5]. Moreover, the rings are rotated with an angle in the range of $\beta_{m} \in[0,2 \pi]$.

It is important to state that the SLL in these TDTD antenna arrays remains similar when the main beam is steered to any direction $\left(\theta_{0 m}, \varphi_{0 m}\right)$ of the space. Figure 6 shows the normalized SLL's values when TDTD arrays focus the main beam for azimuth and elevation planes in steps of ten degrees.

The above behavior of SLL during steering beam can also be achieved for any direction of the main beam such as $\left(\theta_{0}, \varphi_{0}+90^{\circ}\right),\left(\theta_{0}, \varphi_{0}+180^{\circ}\right)$, and $\left(\theta_{0}, \varphi_{0}+270^{\circ}\right)$. Hence, the TDTD antenna arrays give us the opportunity to obtain a high resolution steering with low SLL in the energy pattern in time domain. Now, in order to observe what effect we have in frequency domain after the time-domain synthesis, we calculate the spectrum for the best synthesized geometries. Figures 7 and 8 depict the spectrum in frequency of the far-field waveform in time domain of the RA and UCRA geometries, respectively. The normalized elevation cuts with $\varphi=180^{\circ}$ are shown by sweeping the frequency when the main beam is steered to $\theta_{0}=0^{\circ}$ and $\theta_{0}=45^{\circ}$. The normalization is with respect to the maximum of the pattern in each frequency. A SLL reduction is notably observed for all the frequencies with respect to the nonoptimized or conventional cases. The optimization of the energy patterns in time domain resulted in a good performance in steerable patterns in frequency domain.
Figure 9 depicts the SLL values by varying the frequency for all the array geometries when the main beam is steered into $\varphi_{0}=180^{\circ}$ and $\theta_{0}=45^{\circ}$. The UCRA reached the best SLL reduction from $4 \mathrm{GHz}$ to $10.6 \mathrm{GHz}$ in this illustration. Nonetheless, the RNUCRA, RA, and CCRA have more SLL reduction in the first frequencies rather than the UCRA. This behavior is very similar to that of the main beam when it is steered to other directions.

Table 1 summarized the SLL values for the different TDTD antenna arrays. The performance of those geometries is compared with one another and with respect to the nonoptimized geometries (CUSA and CCRA). The USA has the worst performance in the SLL reduction of the energy pattern because the antenna positions are fixed and the optimum disturbed exciting delays do not provide the capability to reduce more the SLL. The RA and UCRA provide good performances for the SEP with low SLL. There is a slight difference between RA and UCRA geometries in SLL reduction. It is worthy to note that the RA geometry has a simpler beamforming instead of the UCRA geometry due to the use of null disturbed exciting delays. Nonetheless, strictly speaking, the UCRA geometry has a better SLL reduction in time domain and frequency domain. The UCRA and RNUCRA provide a discrepancy of SLL about $\approx 0.2 \mathrm{~dB}$ and SLL $\approx 1 \mathrm{~dB}$, respectively, in the SEP, with respect to the RA. An important guideline is that comparisons among these geometries for a bigger initial fixed aperture and greater numbers of antenna elements are similar. Otherwise, although the mutual coupling was neglected in the synthesis process, these results show the capabilities of different TDTD arrays for a SEP under the same conditions of aperture and number of antenna elements. Finally, Table 2 presents the 


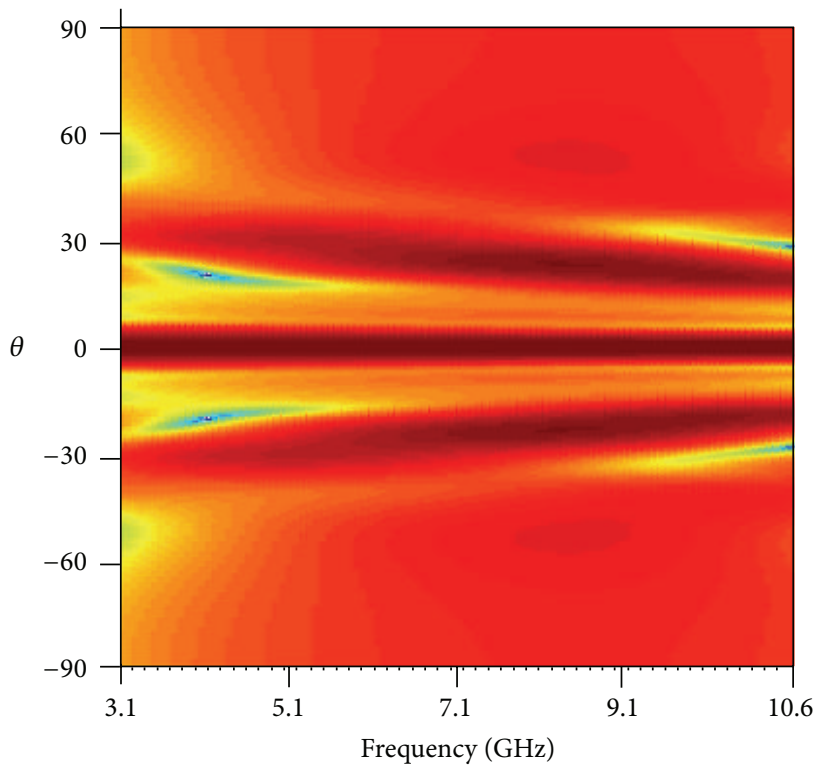

(a)

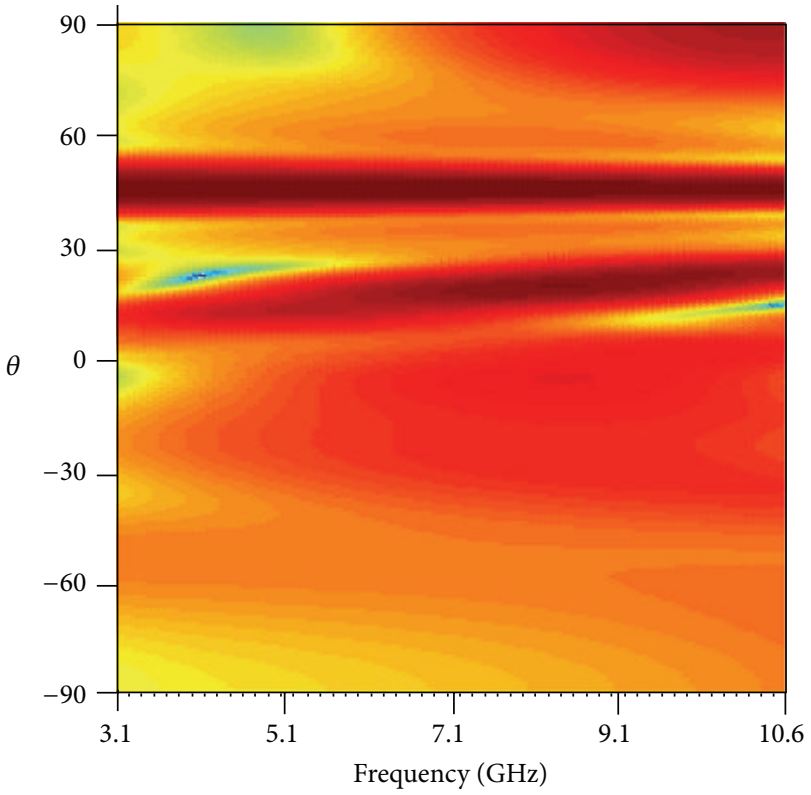

(c)

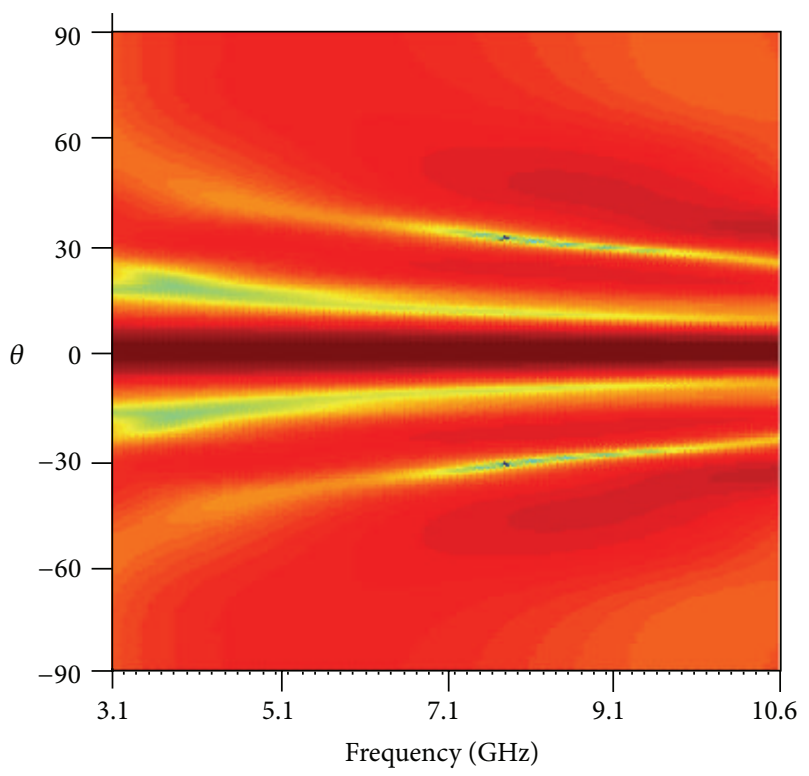

(b)

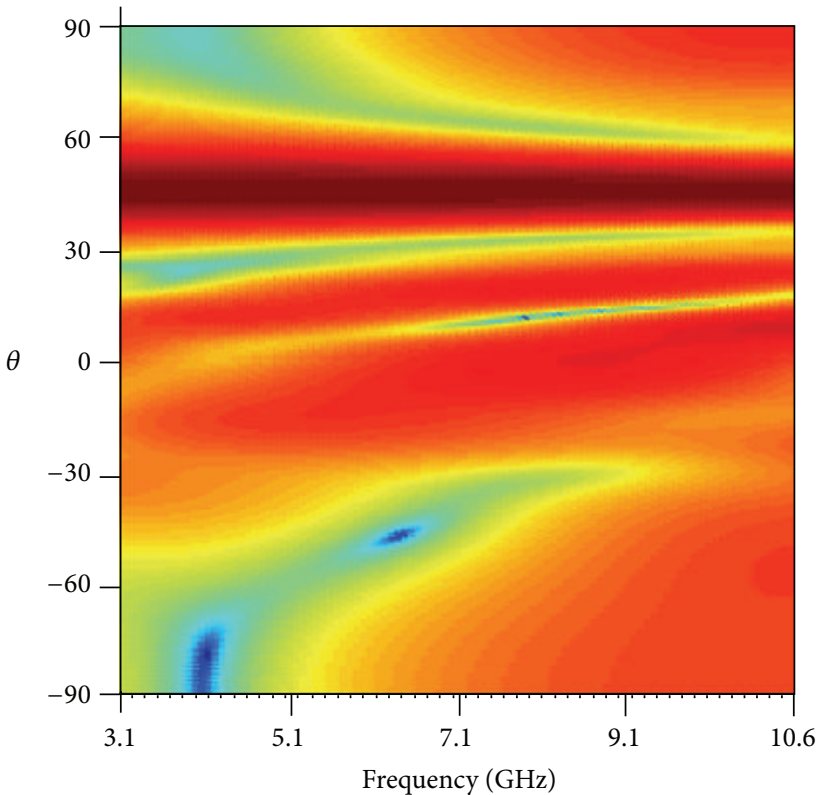

(d)

Figure 7: Spectrum in frequency of (a) CUSA at $\theta=0^{\circ}$, (b) RA at $\theta=0^{\circ}$, (c) CUSA at $\theta=45^{\circ}$, and (d) RA at $\theta=45^{\circ}$.

TABLE 1: SLL values for the TDTD array geometries.

\begin{tabular}{lccc}
\hline Design & SLL in energy pattern in time domain & Maximum SLL in frequency domain & Minimum SLL in frequency domain \\
\hline CUSA & $-4 \mathrm{~dB}$ & $-0.7108 \mathrm{~dB}$ at $8.125 \mathrm{GHz}$ & $-7.8806 \mathrm{~dB}$ at $3.1 \mathrm{GHz}$ \\
CCRA & $-8.3 \mathrm{~dB}$ & $-4.7612 \mathrm{~dB}$ at $8.875 \mathrm{GHz}$ & $-10.0255 \mathrm{~dB}$ at $3.1 \mathrm{GHz}$ \\
USA & $-8.33 \mathrm{~dB}$ & $-1.2898 \mathrm{~dB}$ at $10.6 \mathrm{GHz}$ & $-8.1213 \mathrm{~dB}$ at $6.55 \mathrm{GHz}$ \\
RNUCRA & $-9.54 \mathrm{~dB}$ & $-4.9929 \mathrm{~dB}$ at $10 \mathrm{GHz}$ & $-12.0673 \mathrm{~dB}$ at $3.1 \mathrm{GHz}$ \\
RA & $-10.45 \mathrm{~dB}$ & $-6.1251 \mathrm{~dB}$ at $10.45 \mathrm{GHz}$ & $-10.4407 \mathrm{~dB}$ at $3.25 \mathrm{GHz}$ \\
UCRA & $-10.65 \mathrm{~dB}$ & $-8.3842 \mathrm{~dB}$ at $10.225 \mathrm{GHz}$ & $-10.4315 \mathrm{~dB}$ at $4.9 \mathrm{GHz}$ \\
\hline
\end{tabular}




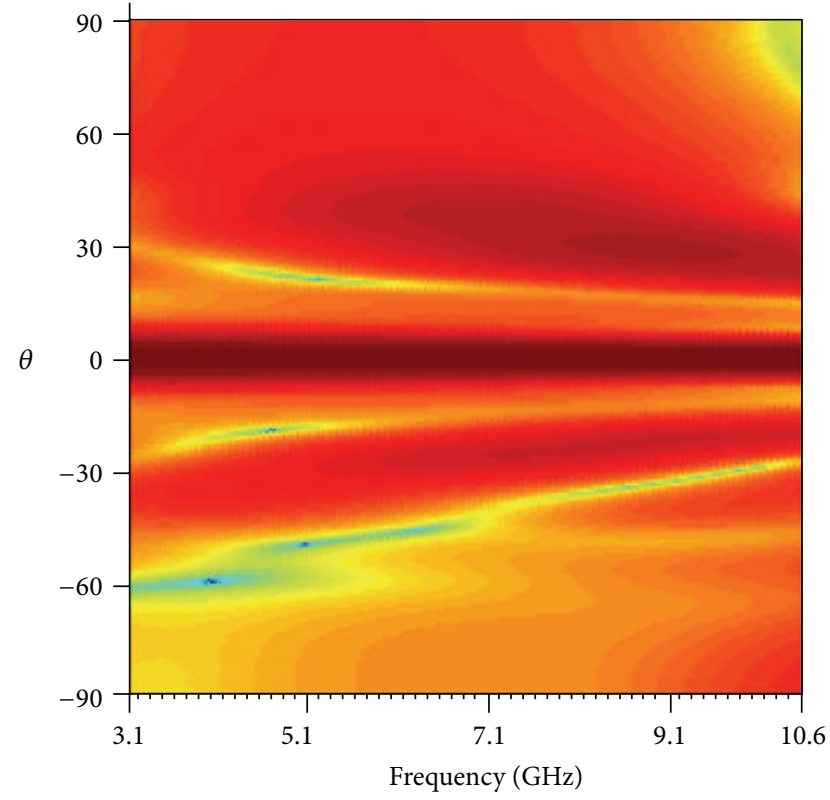

(a)

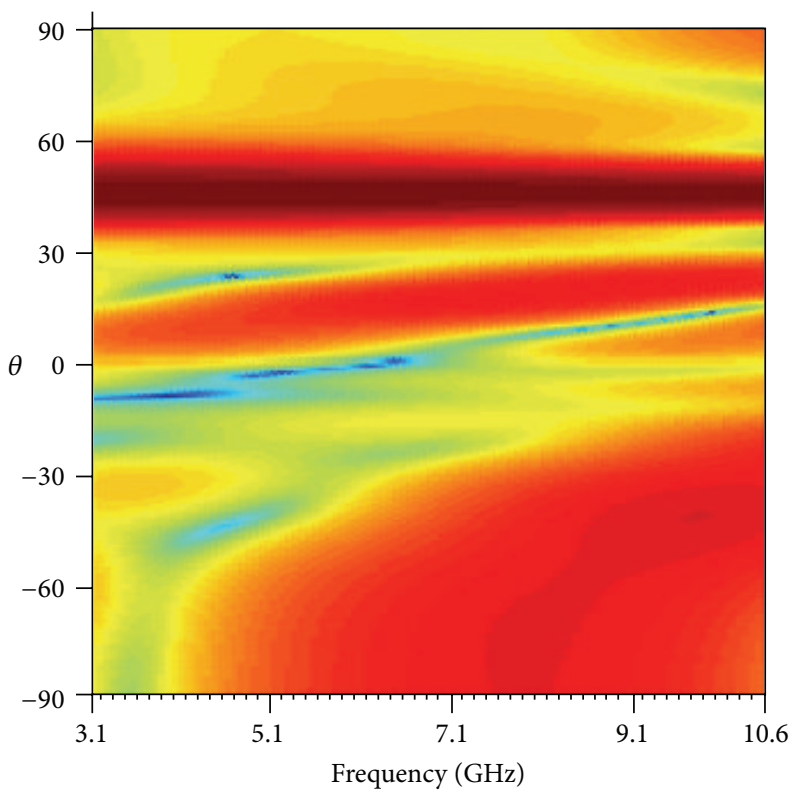

(c)

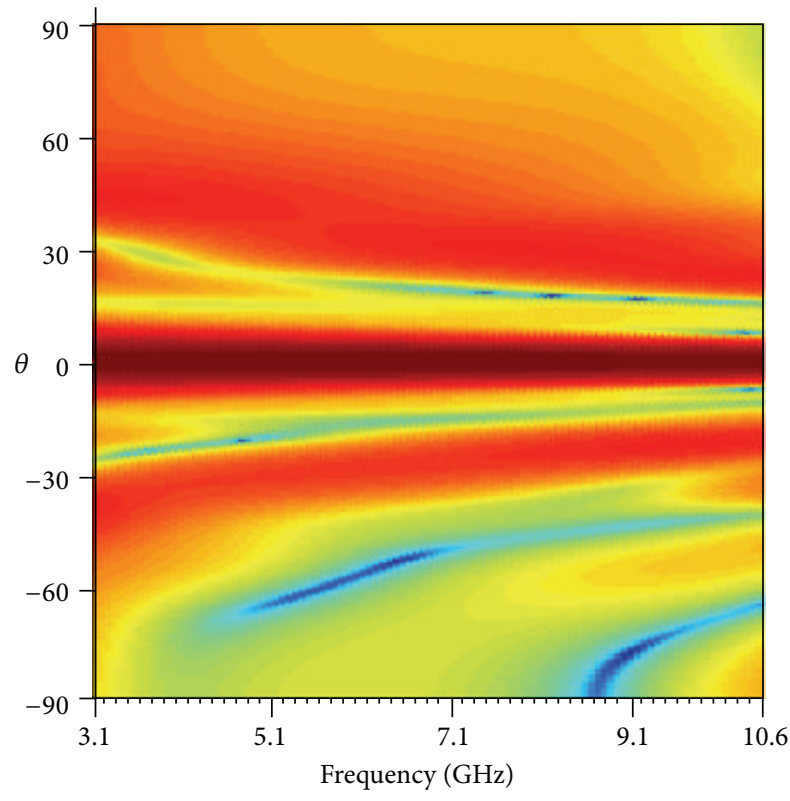

(b)

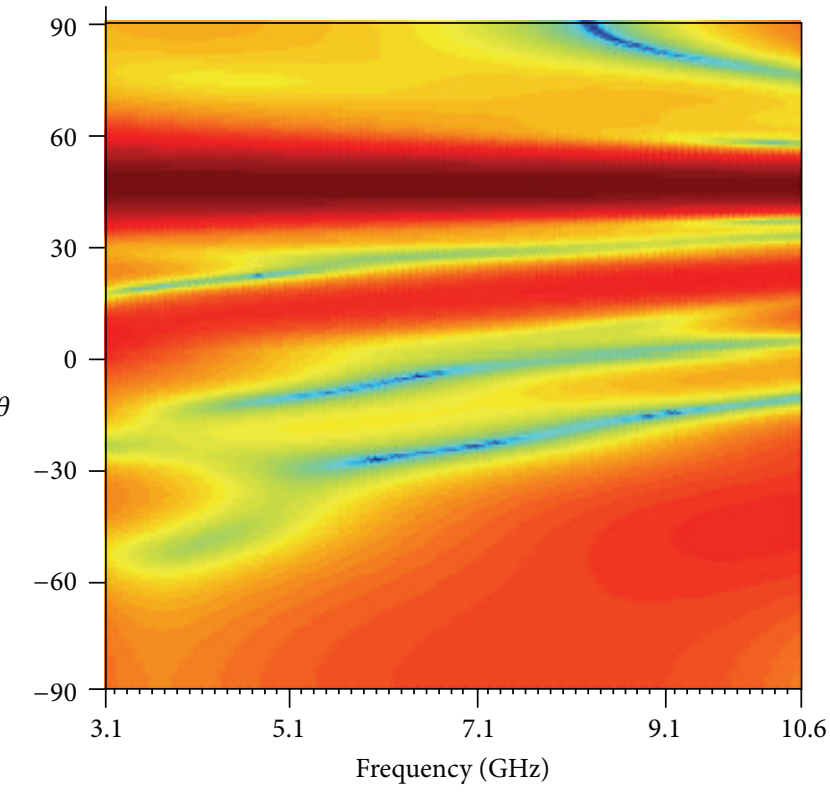

(d)

Figure 8: Spectrum in frequency of (a) CCRA at $\theta=0^{\circ}$, (b) UCRA at $\theta=0^{\circ}$, (c) CCRA at $\theta=45^{\circ}$, and (d) UCRA at $\theta=45^{\circ}$.

design variables after the synthesis for the array geometries; these values may be rounded to an accuracy of $10^{-2}$ without degrading the performance of the geometries.

\section{Conclusions}

This paper addressed the synthesis of energy patterns for different TDTD antenna arrays. The study presented the performance of the optimum values of SLL of energy patterns and their effects in the spectrum in frequency for the best array geometries. The synthesis of arrays in time domain resulted in good performance of all the patterns in frequency even with steering properties. This proposed approach is very useful to design UWB antenna arrays. The TDTD arrays have the capability to steer the beam with low SLL in azimuth and elevation planes for a very high resolution; this can be accomplished by using uniform and nonuniform distributions of disturbed exciting delays and antenna positions. The results showed a better behavior of the UCRA geometry with respect to the other geometries in terms of SLL reduction. And the RA has a very similar performance in the energy pattern but with a simpler beamforming. Future works can be oriented 
TABLE 2: Design variables for the TDTD array geometries.

\begin{tabular}{|c|c|c|c|c|}
\hline & $\tau_{1}$ & $\tau_{2}$ & $\tau_{3}$ & $\tau_{4}$ \\
\hline \multirow[t]{2}{*}{ CUSA } & $0 \mathrm{ps}$ & $0 \mathrm{ps}$ & $0 \mathrm{ps}$ & $0 \mathrm{ps}$ \\
\hline & $\tau_{n 1}$ & $\tau_{n 2}$ & $\tau_{n 3}$ & - \\
\hline \multirow[t]{2}{*}{ CCRA } & $0 \mathrm{ps}$ & $0 \mathrm{ps}$ & $0 \mathrm{ps}$ & - \\
\hline & $\tau_{1}$ & $\tau_{2}$ & $\tau_{3}$ & $\tau_{4}$ \\
\hline \multirow[t]{2}{*}{ USA } & $55.0914 \mathrm{ps}$ & $100.0000 \mathrm{ps}$ & $100.0000 \mathrm{ps}$ & $83.6333 \mathrm{ps}$ \\
\hline & $r_{1}$ & $r_{2}$ & $\beta_{1}$ & $\beta_{2}$ \\
\hline \multirow[t]{2}{*}{ RNUCRA } & $51.9890 \mathrm{~mm}$ & $75.0000 \mathrm{~mm}$ & $3.2700 \mathrm{rad}$ & $6.0967 \mathrm{rad}$ \\
\hline & $\left(x_{1}, y_{1}\right)$ & $\left(x_{2}, y_{2}\right)$ & $\left(x_{3}, y_{3}\right)$ & $\left(x_{4}, y_{4}\right)$ \\
\hline \multirow[t]{2}{*}{ RA } & $76.0423 \mathrm{~mm}, 44.1441 \mathrm{~mm}$ & $149.4227 \mathrm{~mm}, 61.1663 \mathrm{~mm}$ & $25.0000 \mathrm{~mm}, 140.7737 \mathrm{~mm}$ & $41.0591 \mathrm{~mm}, 93.4228 \mathrm{~mm}$ \\
\hline & $\tau_{n 1}$ & $\tau_{n 2}$ & $\tau_{n 3}$ & - \\
\hline UCRA & $50 \mathrm{ps}$ & 50 ps & $47.1491 \mathrm{ps}$ & - \\
\hline
\end{tabular}

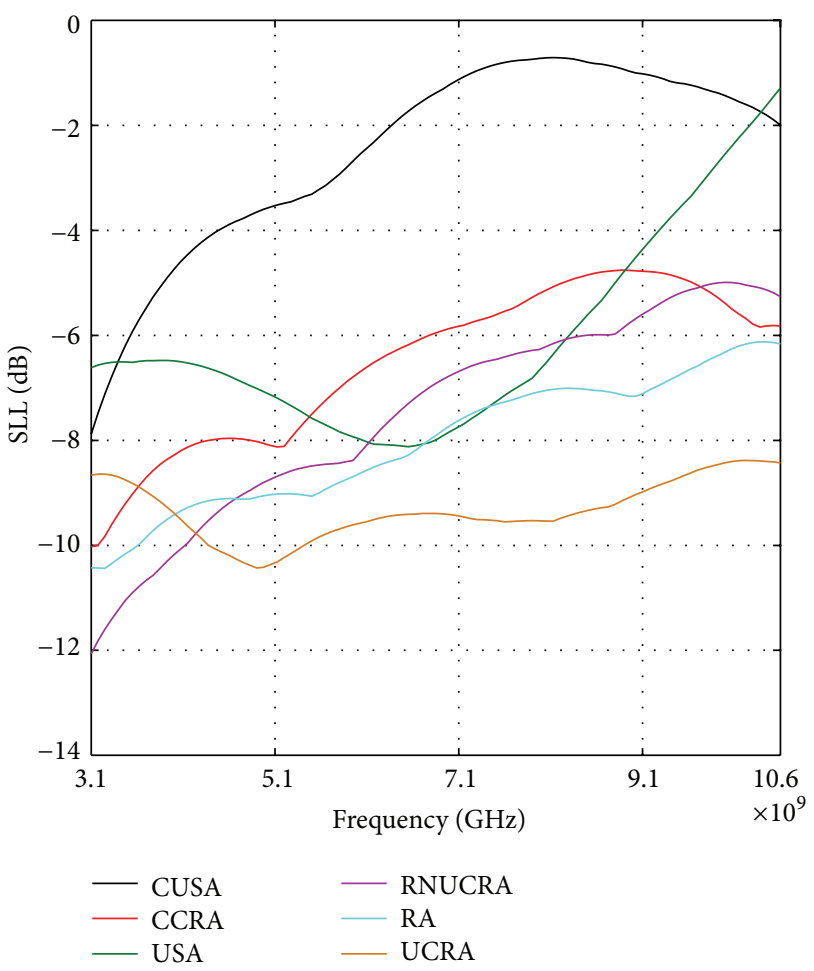

FIgURE 9: SLL values for the TDTD arrays in the frequency.

on the study of these types of arrays by including the bit error rate performance in an UWB system.

\section{Conflict of Interests}

The authors declare that there is no conflict of interests regarding the publication of this paper.

\section{References}

[1] T. W. Barrett, "History of ultra wideband communications and radar: part I, UWB communications," Microwave Journal, vol. 44, no. 1, pp. 22-56, 2001.
[2] A. Shlivinski, "Antenna characterization in the time domain," IEEE Transactions on Antennas and Propagation, vol. 45, no. 7, pp. 1140-1149, 1997.

[3] H. D. Griffiths and A. L. Cullen, "Sidelobe response of antennas to short pulse signals," in Proceedings of the IEEE Radar Conference, pp. 85-90, May 2003.

[4] X. H. Wu, A. A. Kishk, and Z. N. Chen, "A linear antenna array for UWB applications," in Proceedings of the IEEE Antennas and Propagation Society International Symposium and USNC/URSI Meeting, vol. 1A, pp. 594-597, IEEE, July 2005.

[5] S. Chamaani, S. A. Mirtaheri, and M. S. Abrishamian, "Timedomain design of UWB vivaldi antenna array using multiobjective particle swarm optimization," in Proceedings of the 18th Iranian Conference on Electrical Engineering (ICEE '10), pp. 119-123, May 2010.

[6] M. A. Panduro and H. Foltz, "Energy Patterns of UWB antenna arrays with low side lobe level during beam-scanning," in Proceedings of the IEEE International Symposium on Antennas and Propagation (AP-URSI '13), Orlando, Fla, USA, July 2013.

[7] C.-H. Liao, P. Hsu, and D.-C. Chang, "Energy patterns of UWB antenna arrays with scan capability," IEEE Transactions on Antennas and Propagation, vol. 59, no. 4, pp. 1140-1147, 2011.

[8] Y. Xue-Lin, Z. Guang-Fu, H. Jin-Jian, and Y. Nai-Chang, "Study of UWB time-domain antenna array scan," in Proceedings of the International Conference on Microwave and Millimeter Wave Technology (ICMMT '08), vol. 3, pp. 1134-1136, April 2008.

[9] G. Franceschetti, "Timed arrays as new imaging systems," in Proceedings of the 7th European Conference on Antennas and Propagation (EuCAP '13), pp. 142-143, Gothenburg, Sweden, April 2013.

[10] A. Shlivinski, I. E. Lager, and E. Heyman, "Non-uniform array antennas-the time-domain perspective," in Proceedings of the 7th European Conference on Antennas and Propagation (EuCAP '13), pp. 133-137, Gothenburg, Sweden, April 2013.

[11] M. Ciattaglia and G. Marrocco, "Time domain synthesis of pulsed arrays," IEEE Transactions on Antennas and Propagation, vol. 56, no. 7, pp. 1928-1938, 2008.

[12] D. A. Leatherwood, L. E. Corey, R. B. Cotton, and B. S. Mitchell, "Time-domain properties of phased array antennas," in Proceedings of the IEEE International Conference on Phased Array Systems and Technology, pp. 25-28, May 2000.

[13] Y. Qin, C. Liao, and T. Wei, "Application of micro genetic algorithm to optimization of time-domain ultra-wide band antenna 
array," in Proceedings of the International Conference on Microwave and Millimeter Wave Technology (ICMMT '07), pp. 1-4, Builin, China, April 2007.

[14] J. L. Schwartz and B. D. Steinberg, "Ultrasparse, ultrawideband arrays," IEEE Transactions on Ultrasonics, Ferroelectrics, and Frequency Control, vol. 45, no. 2, pp. 376-393, 1998.

[15] G. Marrocco and G. Galletta, "Hermite-rodriguez UWB circular arrays," IEEE Transactions on Antennas and Propagation, vol. 58, no. 2, pp. 381-390, 2010.

[16] R. L. Haupt, "Fitting time delay units in a large wideband corporate fed array," in Proceedings of the IEEE Radar Conference (RADAR '13), pp. 1-4, Ottawa, Canada, April 2013.

[17] Z. Li, P. Papalambros, and J. Volakis, "Antenna optimization using sequential quadratic programming (SQP) algorithms," in Proceedings of the IEEE Antennas and Propagation Society International Symposium, vol. 1, pp. 514-517, Montreal, Canada, July 1997.

[18] Z. Li, P. Papalambros, and J. Volakis, "Antenna optimization using sequential quadratic programming (SQP) algorithms," in Proceedings of the IEEE Antennas and Propagation Society International Symposium, pp. 514-517, IEEE, July 1997.

[19] A. Hammami, R. Ghayoula, and A. Gharsallah, "Planar array antenna pattern nulling based on sequential quadratic programming (SQP) algorithm," in Proceedings of the 8th International Multi-Conference on Systems, Signals and Devices (SSD '11), 7, p. 1, March 2011.

[20] A. Hammami, R. Ghayoula, and A. Gharsallah, "Uniform circular phased arrays synthesis using SQP algorithm," in Proceedings of the 11th Mediterranean Microwave Symposium (MMS '11), pp. 185-188, Hammamet, Tunisia, September 2011.

[21] A. Hammami, R. Ghayoula, and A. Gharsallah, "Design of concentric ring arrays for low side lobe level using SQP algorithm," Journal of Electromagnetic Waves and Applications, vol. 27, no. 7, pp. 807-818, 2013.

[22] H. Sheng, P. Orlik, A. M. Haimovich, L. J. Cimini Jr., and J. Zhang, "On the spectral and power requirements for ultrawideband transmission," in Proceedings of the IEEE International Conference on Communications (ICC '03), vol. 1, pp. 738742, Anchorage, Alaska, USA, May 2003.

[23] H. Kim and Y. Joo, "Fifth-derivative Gaussian pulse generator for UWB system," in Proceedings of the IEEE Radio Frequency Integrated Circuits Symposium (RFIC '05). Digest of Papers, pp. 671-674, IEEE, June 2005.

[24] Z. N. Chen, X. H. Wu, H. F. Li, N. Yang, and M. Y. W. Chia, "Considerations for source pulses and antennas in UWB radio systems," IEEE Transactions on Antennas and Propagation, vol. 52, no. 7, pp. 1739-1748, 2004.

[25] H. Kim, D. Park, and Y. Joo, "All-digital low-power CMOS pulse generator for UWB system," Electronics Letters, vol. 40, no. 24, pp. 1534-1535, 2004.

[26] J. S. McLean, H. Foltz, and R. Sutton, "Pattern descriptors for UWB antennas," IEEE Transactions on Antennas and Propagation, vol. 53, no. 1, pp. 553-559, 2005.

[27] S. Foo and S. Kashyap, "Time-domain array factor for UWB antenna array," Electronics Letters, vol. 39, no. 18, pp. 1304-1305, 2003.

[28] P. T. Boggs and J. W. Tolle, "Sequential quadratic programming," Acta Numerica, vol. 4, pp. 1-51, 1995. 

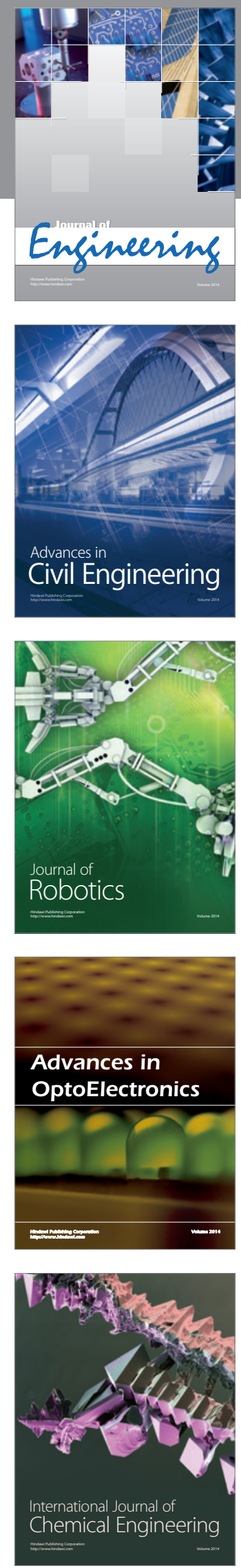

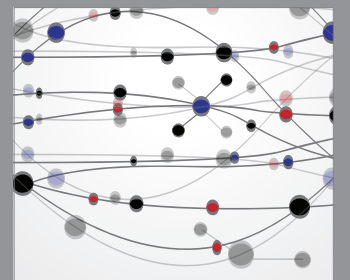

The Scientific World Journal
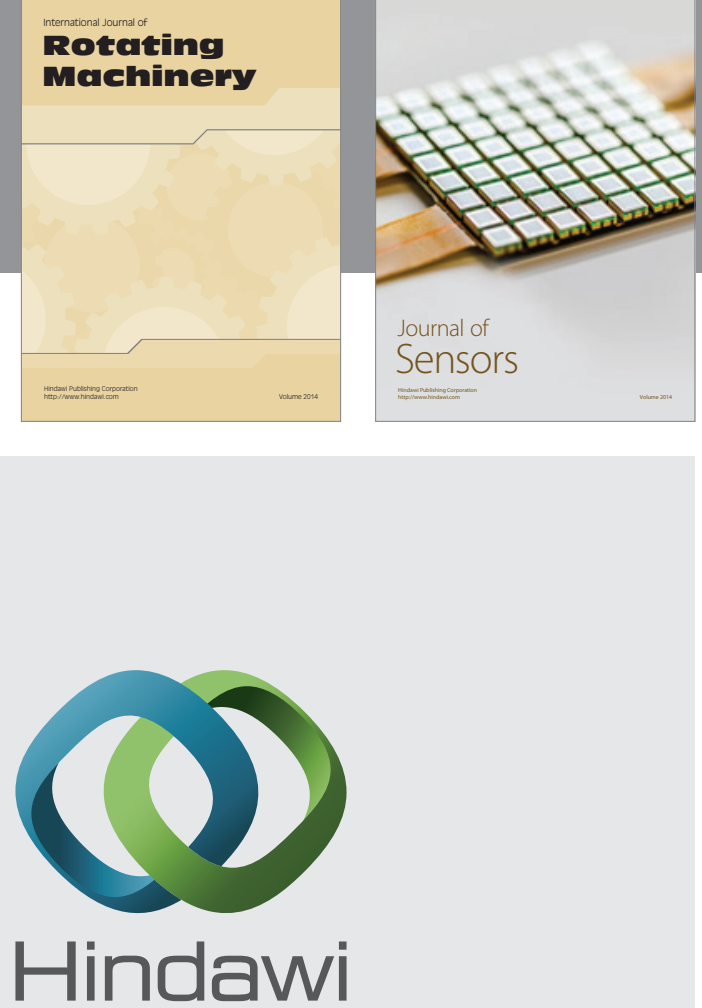

Submit your manuscripts at http://www.hindawi.com
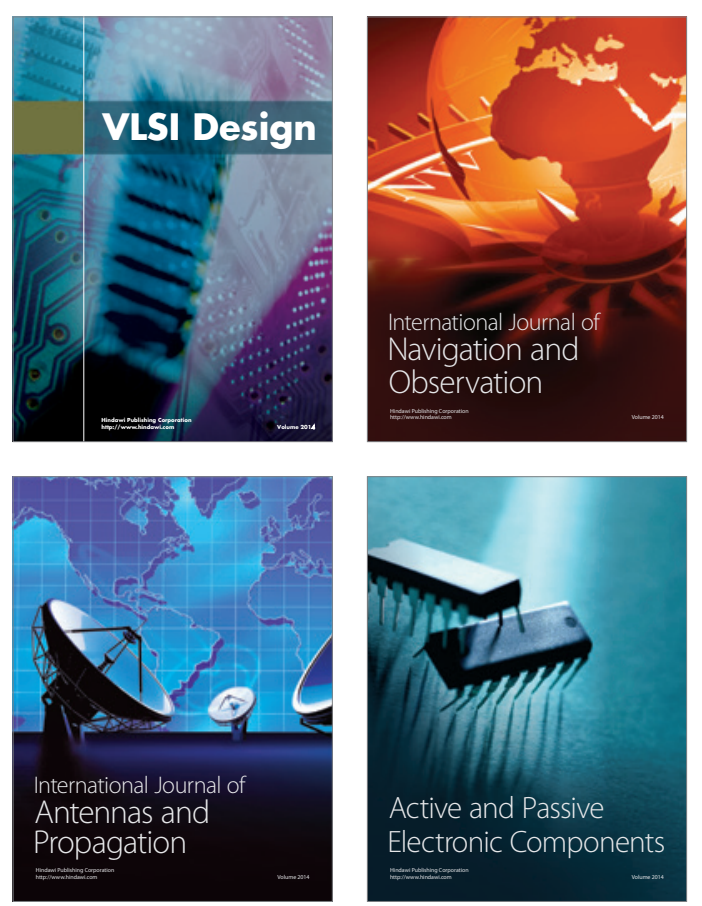
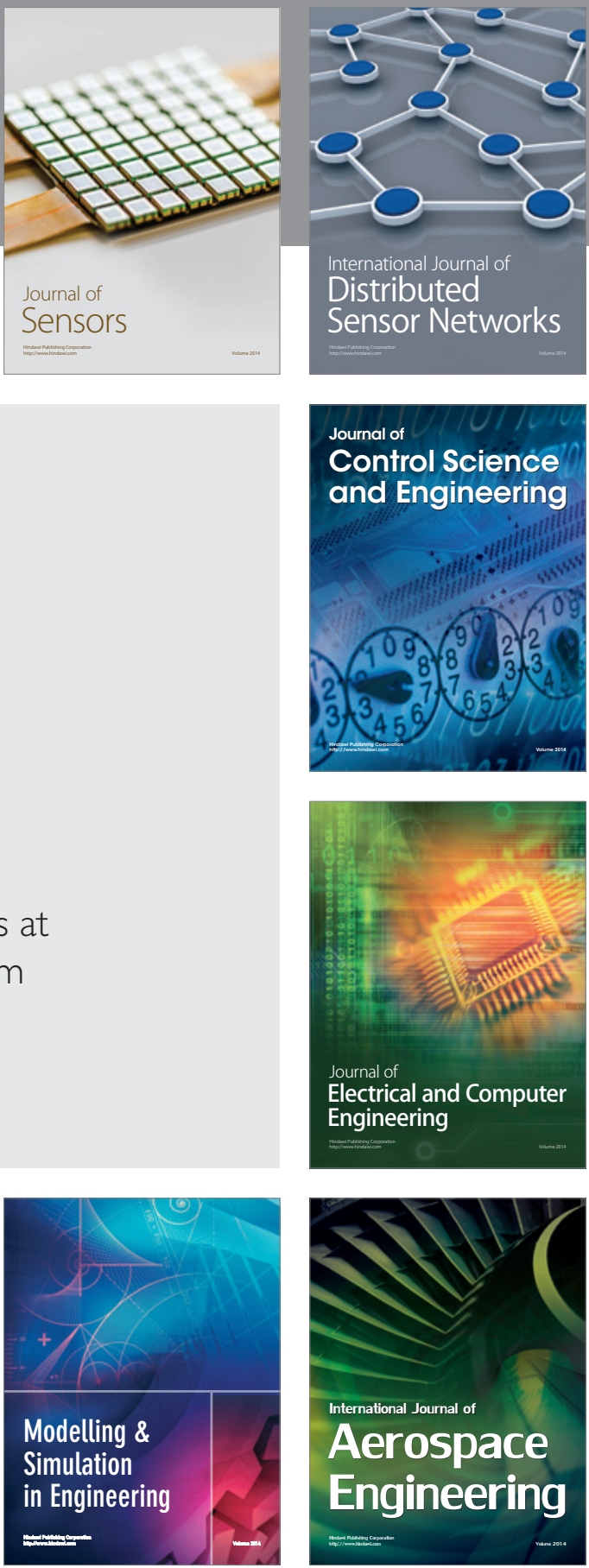

Journal of

Control Science

and Engineering
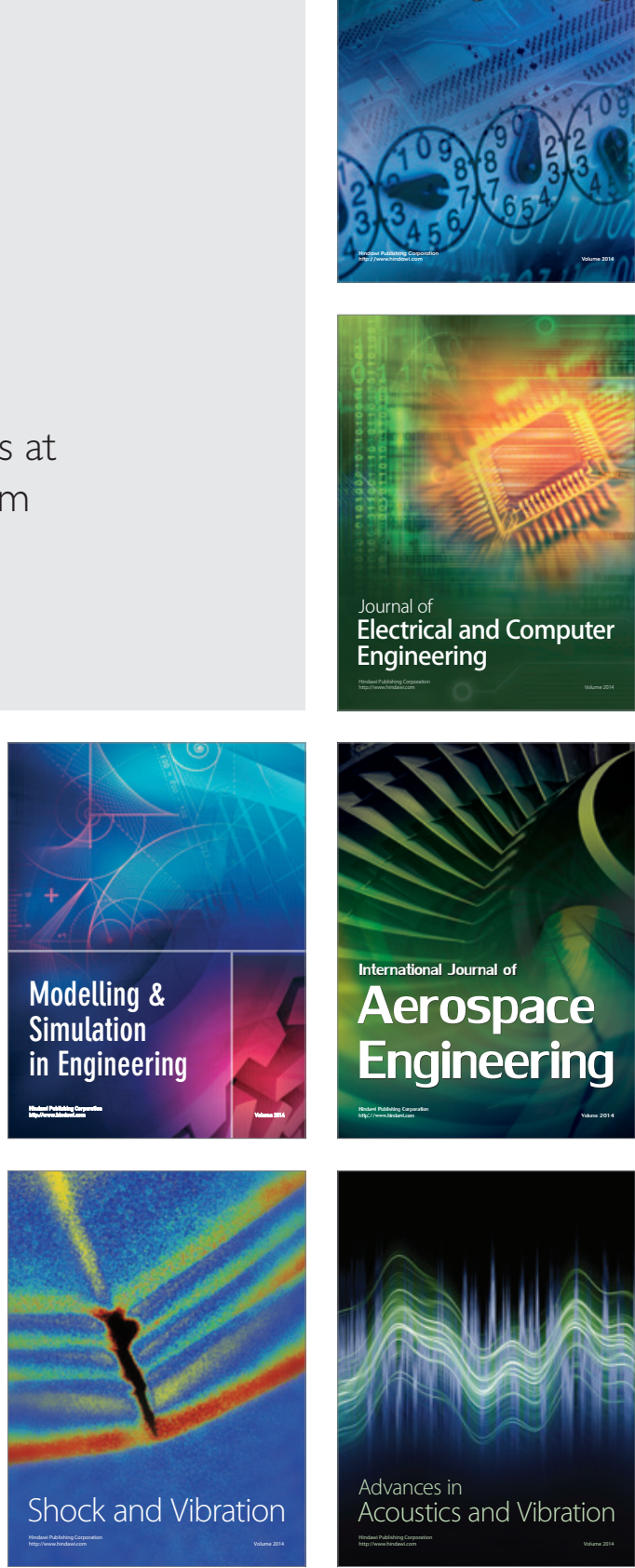